\title{
往复压缩机气量调节控制失稳自愈调控方法研究
}

江志农 ${ }^{1,2}$ 周 超 $^{1}$ 张进杰 ${ }^{1,3}$ 刘雯华 ${ }^{1}$ 王 瑶 ${ }^{1,3}$ 孙 旭 ${ }^{1}$

(1. 北京化工大学压缩机技术国家重点实验室压缩机健康智能监控中心 北京 100029 ;

2. 北京化工大学高端机械装备健康监控与自愈化北京市重点实验室 北京 100029 ;

3. 压缩机技术国家重点实验室(压缩机技术安徽省实验室) 合肥 230031)

摘要: 针对往复压缩机无级气量调节常见的调控失稳故障, 构建包含压缩机、管道、缓冲罐、执行机构等部件的无级气量调 节多系统耦合控制模型, 仿真往复压缩机无级气量调节动态特性。以控制系统输出的脉宽调制控制信号作为输入, 以压缩机 各级排气压力作为输出, 研究执行机构动态响应和控制系统调控参数对气量调节结果影响的规律; 进一步针对执行机构性能 参数变化导致气量调节控制失稳的问题，利用 BP 神经网络构建多参数负荷动态反馈模型实现调控失稳故障诊断和故障类型 识别。基于故障异常类型识别结果提出一种调控参数自适应优化补偿的自愈调控方法。试验结果表明, 提出的自愈调控方法 可在失稳故障发生后主动施加调控参数补偿量, 使得气量调节系统恢复到正常状态, 实现故障在线自愈。

关键词: 往复压缩机; 调控失稳故障; 自愈调控; BP 神经网络

中图分类号: TH457; TB126

\section{Research on Self-healing Control Method of Reciprocating Compressor Capacity Control Instability}

\author{
JIANG Zhinong ${ }^{1,2}$ ZHOU Chao ${ }^{1}$ ZHANG Jinjie ${ }^{1,3}$ LIU Wenhua ${ }^{1}$ WANG Yao ${ }^{1,3}$ SUN Xu ${ }^{1}$
}

(1. Compressor Health and Intelligent Monitoring Center of National Key Laboratory of Compressor

Technology, Beijing University of Chemical Technology, Beijing 100029;

2. Beijing Key Laboratory of Health Monitoring Control and Fault Self-recovery for High-end

Machinery, Beijing University of Chemical Technology, Beijing 100029;

3. State Key Laboratory of Compressor Technology (Anhui Provincial Laboratory of

Compressor Technology), Hefei 230031)

\begin{abstract}
For the common regulation instability fault of reciprocating compressor stepless capacity regulation system, a multi-system coupling control model of stepless capacity regulation system which includes compressor, pipeline, buffer tank, actuator and so on, is built to simulate the dynamic characteristics of reciprocating compressor stepless capacity regulation system. By using the pulse width modulation control signal from the control system as input and the compressor exhaust pressure of every stage as output. The law of the dynamic response of the actuator and the influence of the control parameters in control system on the capacity regulation results are studied. Further, for the problem of capacity control instability caused by the change of actuators' performance parameters, a multi-parameter load dynamic feedback model is built by using BP neural network to realize the regulation instability fault diagnosis and fault type identification. Based on the results of abnormal fault type recognition, a self-healing control method based on adaptive optimal compensation of control parameters is proposed. The experimental results indicated that the proposed self-healing control method can automatically apply the compensation of control parameters after the instability fault, so that the capacity regulation system returned to the normal state, and the fault self-healing can be realized on-line.
\end{abstract}

Key words: reciprocating compressor; regulatory instability failure; self-healing control; BP neural network

* 国家重点研发计划(2016YFF0203305)、中央高校基本科研业务费专项资金资 助(JD1912)和压缩机技术国家重点实验室(压缩机技术安徽省实验室)开放基 金(SKL-YSJ201808)资助项目。20191104 收到初稿, 20200730 收到修改稿 


\section{0 前言}

往复式压缩机作为一种容积式压缩机, 被广泛 应用于石油、化工、炼油、采气、注气、输气等领 域。在许多往复压缩机的运用领域中, 由于工况变 动, 如进气量不足、排气需求降低等, 都需要进行 气量调节。基于部分行程顶开进气阀原理的往复压 缩机流量调节方法是利用执行机构精确控制吸气阀 动作, 按实际需要的气量要求延迟气阀关闭。如在 $2900 \mathrm{~kW}$ 的 4M50 型活塞压缩机上安装了气量调节 系统, 气量调节到 40\% 60\%负荷时, 每小时节电 达 $1200 \mathrm{~kW}$ ，一年正常运转可节约人民币 500 多万 元, 节能效果十分明显 ${ }^{[1]}$ 。

针对气量调节系统的研究主要集中在变负荷调 节工况下压缩机热、动力学特性, 以及变负荷工况对 机组性能的影响 ${ }^{[2-3]}$ 。随着气量调节的广泛应用 ${ }^{[4]}$, 由 于压缩机及附属系统的复杂性, 气量调节系统无法适 合所有压缩机, 执行机构、液压系统及控制硬件等经 常发生故障 ${ }^{[5-8]}$, 系统可靠性及安全性亟需保证。研 究人员针对实际应用问题, 优化分析并设计 ${ }^{[9-11]}$, 针 对气量调节系统研究内容概述如表 1 所示。研究人员 针对实际应用问题, 主要集中在故障分析上, 而且对 于故障的处理方式多为传统的手动补偿及停机检修, 并未研究在故障状态下如何在线自愈, 无法避免停车 损失, 维修成本居高不下, 并且系统过于依赖人工操 作，系统安全性及可靠性仍没有提高。

\section{表 1 气量调节系统研究内容概述}

\begin{tabular}{cccc}
\hline 文献编号 & 压缩机动力特性 & 执行机构故障 & 优化分析 \\
\hline$[2]$ & $\sqrt{ }$ & & \\
{$[3]$} & $\sqrt{ }$ & $\sqrt{ }$ & \\
{$[5]$} & & $\sqrt{ }$ & \\
{$[6]$} & & $\sqrt{ }$ & \\
{$[7]$} & $\sqrt{ }$ & $\sqrt{ }$ \\
{$[8]$} & & & $\sqrt{ }$ \\
{$[9]$} & & & $\sqrt{ }$ \\
{$[10]$} & & & \\
{$[11]$} & & & \\
\hline
\end{tabular}

近年来, 国内外越来越多的学者将自愈调控方 法应用于设备故障领域中。针对天文望远镜驱动系
统, LI 等 ${ }^{[12]}$ 提出了一种基于人工智能的专家系统故 障诊断和无缝自修复方案; GOMEZ-ANDRADES 等 ${ }^{[13]}$ 提出一种基于模糊逻辑的 LTE 网络故障自动 识别系统和修复方案; BUTLERPURRY 等 ${ }^{[14]}$ 提出 了一种用于舰船电源系统服务恢复的自动自修复策 略; 刘凯等 ${ }^{[15]}$ 提出了一种基于启发式规则和人工免 疫的配电网自愈方法。故障自愈调控技术是解决实 际工程中此类问题的有效手段, 通过智能决策和主 动控制方法在运行中抑制和消除故障, 确保机器平 稳可靠运行，实现机器系统故障自愈化 ${ }^{[16]}$ 。

本文针对往复压缩机气量控制失稳的问题, 通 过理论分析失稳的故障类型及关键参数, 构建包含 压缩机、管道、缓冲罐、执行机构等部件的无级气 量调节多系统耦合仿真模型, 通过多参数动态反馈 优化模型, 主动施加控制力消除故障, 提出一种气 量调控失稳的自愈调控方法, 实现气量调节系统在 失稳故障状态下在线自愈功能, 避免传统停机检修 造成的无法连续生产、维修及生产损失大、系统可 靠性及安全性低等问题。

\section{1 多系统耦合控制模型}

往复压缩机增上无级气量调节系统后, 依靠执 行机构控制吸气阀启闭时间实现排气量调节。由于 执行机构及控制阀的高频动作, 系统运动部件易产 生疲劳、磨损等劣化现象, 弹簧疲劳、控制阀参数 偏移等故障经常发生, 导致系统失稳, 严重时需要 停机检修。为了制定自愈方案, 需要得到实际负荷 与设计负荷的偏差, 根据负荷偏差值计算控制参数 的调整量。需要建立压缩机、管道、缓冲罐、执行 机构多系统耦合数学模型研究排气压力与失稳参数 的关系。然后通过动态反馈模型, 将不同故障下的 压力、温度、排气量作为输入, 得到实际负荷的输 出, 为制定自愈方案奠定基础。无级气量调节系统 失稳故障及数学模型拓扑图如图 1 所示。

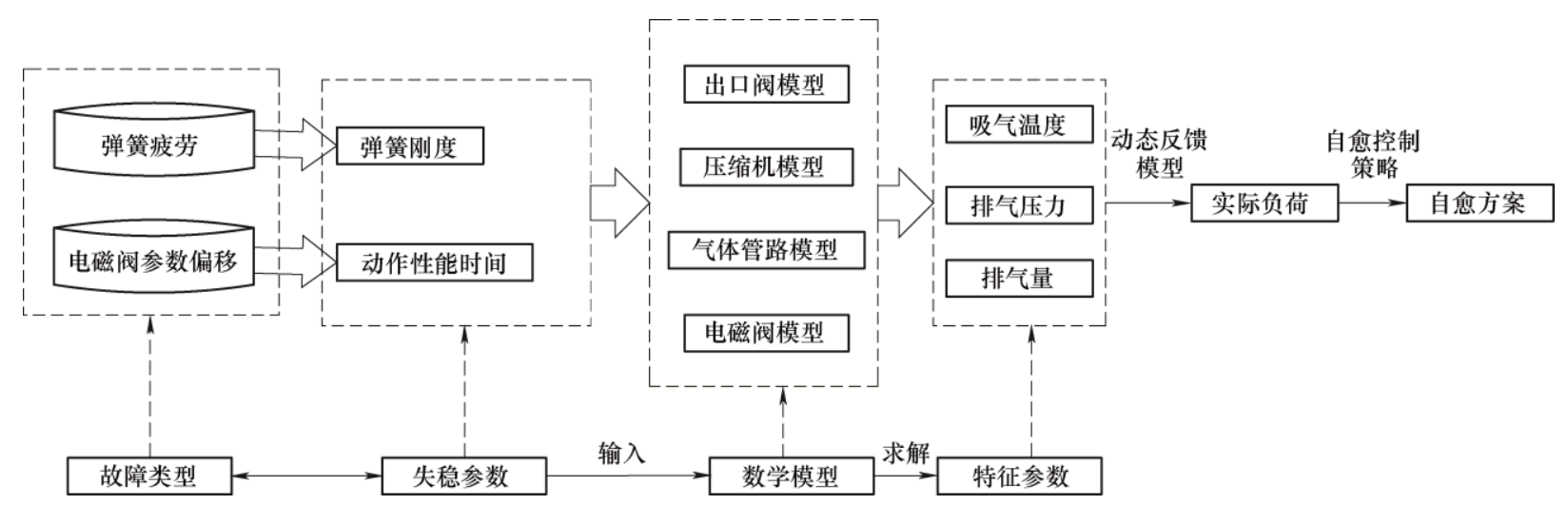

图 1 失稳故障类型及数学模型拓扑 


\section{1 压缩机模型}

以压缩机气缸作为控制容积, 建立往复压缩机 缸内气体热力学变化数学模型, 得到压缩机气缸吸 排气流量的变化特性, 根据由热力学第一定律建立 描述气缸压力 $P_{c y}$ 变化的表达式为

$$
\begin{gathered}
\frac{\mathrm{d} p_{c y}}{\mathrm{~d} \theta}=\frac{1}{V_{c y}(\theta)}\left(c_{i}{ }^{2} \frac{\mathrm{d} m_{c i n}}{\mathrm{~d} \theta}-c_{c y}{ }^{2} \frac{\mathrm{d} m_{c d}}{\mathrm{~d} \theta}-\gamma p_{c y} \frac{\mathrm{d} V_{c y}}{\mathrm{~d} \theta}\right) \\
V_{c y}(\theta)=V_{c 0}+S_{c y} x_{c y}(\theta) \\
\frac{\mathrm{d} V_{c y}(\theta)}{\mathrm{d} \theta}=S_{c y} \frac{\mathrm{d} x_{c y}(\theta)}{\mathrm{d} \theta}
\end{gathered}
$$

式中, $\theta=\omega t$ 表示曲轴相较于外止点转动的角度, $\omega$ 为曲轴转动的角速度, $V_{c y}(\theta)$ 表示气缸容积, $V_{c 0}$ 表 示气缸余隙容积, $S_{c y}$ 表示活塞面积, $x_{c y}(\theta)$ 活塞位 移; $\frac{\mathrm{d} V_{c y}}{\mathrm{~d} \theta}$ 表示曲轴转动单位角度时活塞运动的线速 度; $\frac{\mathrm{d} m_{c i n}}{\mathrm{~d} \theta}$ 和 $\frac{\mathrm{d} m_{c d}}{\mathrm{~d} \theta}$ 分别表示曲轴转动单位角度时通 过进气阀流进和通过排气阀流出气缸的质量流量, $\gamma=1.4$ 表示理想气体的比热比; $c_{i}^{2}=\gamma R T_{i}$ 表示在进 气腔中气体声速的平方, $c_{c y}{ }^{2}=\gamma R T_{c y}$ 表示在气缸中 气体声速的平方, $R$ 表示理想的气体常数。

由于电液执行机构的介入, 吸气阀片动作过程 由自动阀方式变为强制阀方式, 排气阀仍然为自动 阀。吸气阀根据气阀两侧的压力差进行开闭动作, 阀片运动过程受气体压差、执行机构作用力、复位 弹簧力、阀片弹簧力的综合作用, 具体表示为

$$
M_{s v} \omega \frac{\mathrm{d}^{2} x_{s v}}{\mathrm{~d} \theta^{2}}+c_{s s} \omega \frac{\mathrm{d} x_{s v}}{\mathrm{~d} \theta}+k_{s v} x_{s v}-F_{h u}=\sum f_{s v}
$$

式中, $F_{h u}$ 为电液执行器输出液压力; $M_{S v}$ 为吸气阀 和卸荷器总的运动质量。 $\frac{\mathrm{d}^{2} x_{s v}}{\mathrm{~d} \theta^{2}}, \frac{\mathrm{d} x_{s v}}{\mathrm{~d} \theta}$, 和 $x_{s v}$ 分别 表示进气阀片的加速度、速度和位移; $c_{s s}$ 表示阻 尼系数, $k_{s v}$ 表示总弹簧刚性系数, $\sum f_{s v}$ 表示作用 在阀片上的合力。

通过吸气阀质量流量 $\frac{\mathrm{d} m_{c i n}}{\mathrm{~d} \theta}$ 和排气阀的质量流 量 $\frac{\mathrm{d} m_{c d}}{\mathrm{~d} \theta}$ 可以表示为

$$
\begin{aligned}
& \frac{\mathrm{d} m_{c i n}}{\mathrm{~d} \theta}=C_{i} A_{f s} \frac{1}{\omega} \sqrt{2 \rho_{c y}\left|p_{i}-p_{c y}\right|} \\
& \frac{\mathrm{d} m_{c d}}{\mathrm{~d} \theta}=C_{d} A_{f d} \frac{1}{\omega} \sqrt{2 \rho_{c y}\left|p_{d}-p_{c y}\right|}
\end{aligned}
$$

式中, $A_{f s}$ 表示吸气阀通流面积; $C_{i}$ 表示吸气流量
系数; $p_{i} 、 p_{d}$ 表示吸气和排气压力; $A_{f d}$ 表示排气 阀通流面积; $C_{d}$ 表示排气流量系数, 由气阀通道的 形状等因数决定, 可查表获得; $\rho_{c y}$ 表示气缸内气体 的密度。

\section{2 气体管路模型}

气体在压缩机各级进排气与缓冲罐之间的管路 之中传递, 需要建立气体管路模型分析气体压力和 温度, 采用多变方程进行建模分析, 管路模型图如 图 2 所示。管路长度设为 $L$, 管径为 $D$ 。

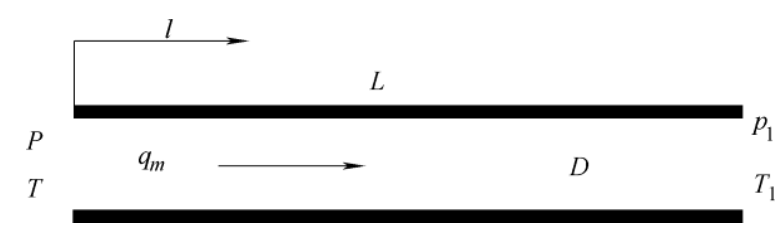

图 2 管路的简化物理模型

管路中，气体的质量流量变化方程如下。

$$
\dot{m}_{\text {tout }}(L, t)= \begin{cases}0 & t<L / c \\ \exp \left(-\frac{R_{t} R T}{2 p} \frac{L}{c}\right) q\left(t-\frac{L}{c}\right) & t>L / c\end{cases}
$$

式中, $\dot{m}_{\text {tout }}(L, t)$ 表示通过管路流出的质量流量; $q(t)$ 表示管路入口流量; $R_{t}$ 表示管路阻抗; $c$ 表示管路 内气流速度; $P, T$ 分别表示入口压力和温度。

\section{3 执行机构动态特性数学模型}

为了简化分析电液执行器开启过程的数学模 型, 采用一个固定开口液阻模拟高速换向阀的阀口 工作特性 ${ }^{[17]}$, 可以得到电液执行机构的动态特性模 型为

$$
\left\{\begin{array}{l}
Q_{h}(u)=C_{h} A_{h} u(\mathrm{t}) \sqrt{\frac{2\left(P_{\text {oil }}-P_{A}\right)}{\rho_{\text {oil }}}}+ \\
C_{h} A_{h}(u-1) \sqrt{\frac{2}{\rho_{\text {oil }}} P_{A}} \\
Q_{h}(u)=A_{A} \dot{x}+C_{l} P_{A} \\
P_{A} A_{A}-K_{f}\left(x+x_{0}\right)=m_{A} \ddot{x}+B \dot{x}
\end{array}\right.
$$

式中, $Q_{h}$ 表示高速换向阀控制口液压油流量; $C_{h}$ 表 示阀口流量系数; $A_{h}$ 表示高速换向阀阀口通流面 积; $P_{\text {oil }}$ 表示油站供油压力; $P_{A}$ 表示液压缸内压力; $\rho_{\text {oil }}$ 表示液压油密度; $A_{A}$ 表示油缸活塞面积; $x$ 表 示负载位移; $C_{l}$ 表示油缸内泄露系数; $K_{f}$ 表示弹簧 刚度; $B$ 表示油缸阻尼系数; $x_{0}$ 表示负载弹簧预压 缩量; $m_{A}$ 表示油缸活塞及负载等效质量; $u=\{0,1\}$ 为电液执行器控制信号, $u=1$ 表示控制信号高电平 使得电液执行机构开启和维持打开的过程, $u=0$ 表 示控制信号低电平执行机构关闭的过程。 


\section{4 出口阀流量模型}

往复压缩机末级排气缓冲罐有一个单向阀与工 艺管路连接, 当缓冲罐的压力大于阀后压力时, 缓 冲罐中的气体通过单向阀向外排气。通过阀口面积 $A_{v}$ 的质量流量的标准方程可表示为

$$
\dot{m}_{v}\left(P_{u}, P_{d}\right)= \begin{cases}C_{f} A_{v} C_{1} \frac{P_{u}}{\sqrt{T}} & \frac{P_{d}}{P_{u}} \leqslant P_{c r} \\ C_{f} A_{v} C_{2} \frac{P_{u}}{\sqrt{T}}\left(\frac{P_{d}}{P_{u}}\right)^{1 / k} \sqrt{1-\left(\frac{P_{d}}{P_{u}}\right)^{(k-1) / k}} & \frac{P_{d}}{P_{u}}>P_{c r}\end{cases}
$$

式中, $\dot{m}_{v}$ 表示流过阀门孔口的质量流量; $P_{u}$ 为阀前 压力; $P_{d}$ 表示阀后压力; $k$ 为理想气体的比热比, 临界值 $P_{c r}=0.528$ 。 $C_{1}$ 和 $C_{2}$ 分别表示比热比相关的 调节系数; $C_{f}$ 是流量系数, 一般取值 $0.8 \sim 0.85$ 。

\section{5 缓冲罐压力模型}

对于可变控制体积的气腔, 其腔内压力的变化 可由三个重要的方程求得, 根据质量连续性方程、 理想气体状态方程和能量方程, 可得到控制容积 $V$ 内压力动态方程的统一形式。

$$
\dot{P}=\frac{R T}{V}\left(\alpha_{\text {in }} \dot{m}_{\text {in }}-\alpha_{\text {out }} \dot{m}_{\text {out }}\right)-\alpha \frac{P}{V} \dot{V}
$$

式中, $\alpha, \alpha_{i n}$ 和 $\alpha_{o u t}$ 表示气体流入和流出的系数, 其取值取决于实际过程的传热过程, 其取值范围在 1 和 $k$ 之间。对于往复压缩机各级进排气缓冲罐, 体积保持不变且罐内气体可通过管壁与外界进行充 分地热交换, 可近似为等温过程, 即 $\alpha_{i n}=\alpha_{o u t}=1$, 缓冲罐压力可表示为

$$
\dot{P}_{b u f f}=\frac{R T}{V_{\text {buff }}}\left(\dot{m}_{\text {in_buff }}-\dot{m}_{\text {out_buff }}\right)
$$

式中, $\dot{P}_{b u f f}$ 表示缓冲罐内压力变化; $V_{b u f f}$ 表示缓冲 罐体积; $\dot{m}_{i n_{-} b u f f}$ 和 $\dot{m}_{\text {out_buff }}$ 分别表示流进和流出缓冲 罐的气体质量流量。

考虑缓冲罐与压缩机气缸之间的管路的影响, 假定一级气缸与级间缓冲罐的管路长度为 $L_{1}$, 二级 气缸与级间缓冲罐的管路长度为 $L_{2}$, 二级气缸与排 气缓冲罐的管路长度为 $L_{3}$ 。二级缓冲罐出口阀后的 背压为 $P_{\mathrm{dd}}$, 级间缓冲罐和排气缓冲罐体积分别为 $V_{1}$ 和 $V_{2}$ 。联立式(1) (11) 建立两级压缩机多系统耦 合数学模型。

$$
\left\{\begin{array}{l}
\dot{p}_{1}=\frac{R T_{1}}{V_{1}}\left(\dot{m}_{\text {lout }}\left(L_{1}, t\right)-\dot{m}_{2 i n}\left(L_{2}, t\right)\right. \\
\dot{p}_{2}=\frac{R T_{2}}{V_{2}}\left(\dot{m}_{2 o u t}\left(L_{3}, t\right)-\dot{m}_{v}\left(P_{2}, P_{d d}\right)\right)
\end{array}\right.
$$

式中, $P_{1}$ 为级间缓冲罐压力, $P_{2}$ 为末级排气缓冲罐 压力。

\section{Simulink 仿真模型及结果分析}

为研究失稳参数对气量调节结果的影响规律, 采 用一台 $2 \mathrm{D}$ 双作用两级往复空气压缩机、排气管路和 排气缓冲罐作为研究对象, 利用所建立的多系统耦合 数学模型, 使用 Matlab 仿真模拟器 Simulink 进行仿真 试验, Simulink 仿真框图如图 3 所示, 为压缩机气量 系统失稳故障的自愈控制提供理论基础和支撑。

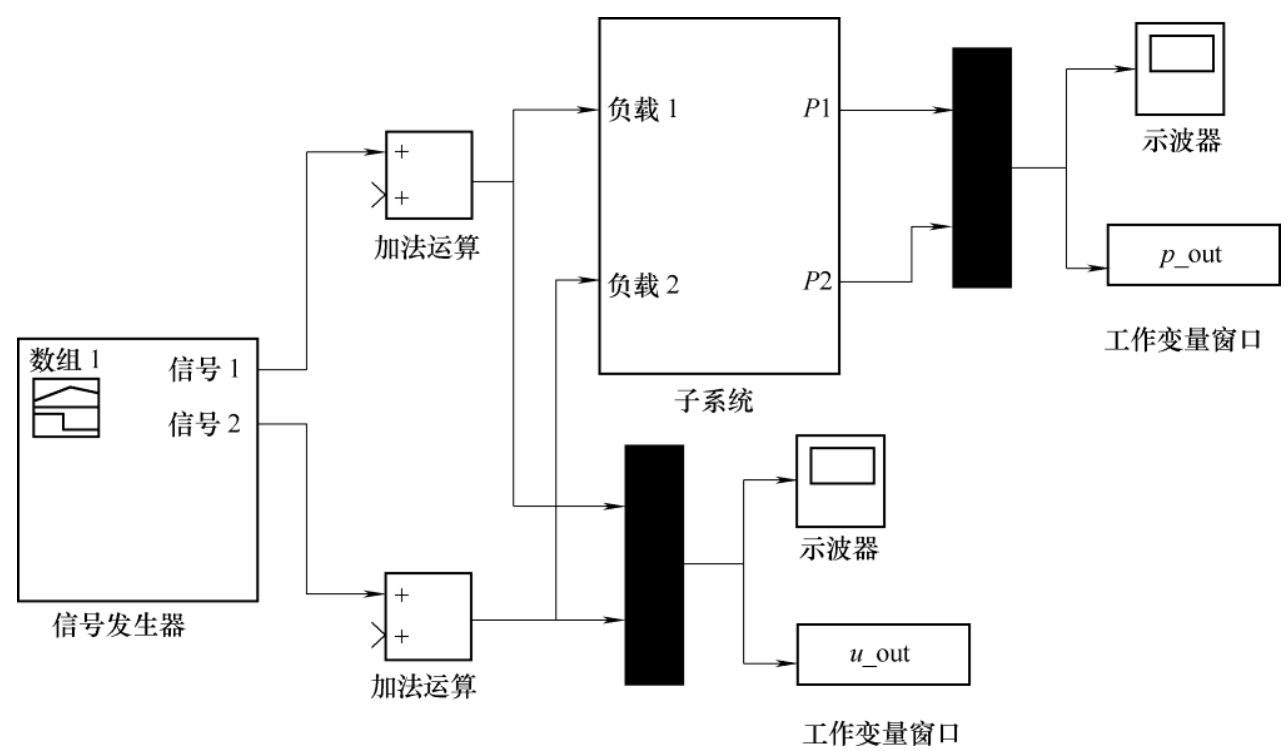

图 3 Simulink 仿真框图

由于执行机构复位弹簧在高频动作下易发生疲 劳, 弹簧刚度减小, 造成执行机构动作变慢系统失
稳; 由于控制阀的诸多零件处于高频切换工作状态, 例如线圈、衔铁、弹簧等易发生磨损，影响控制阀 
动作性能时间, 控制阀动态响应时间提前或滞后造 成系统失稳。因此分别研究不同复位弹簧刚度和控 制阀动态响应时间参数对气量调节的影响。输入信 号为 $u_{1}$ (弹簧刚度)和 $u_{2}$ (控制阀动作性能时间), 输出 信号为压缩机排气压力 $P_{1}$ 和 $P_{2}$, 为了分析不同失稳 故障对气量调节的影响, 仿真信号如表 2 所示, 由于仿真数据较多, 截取部分仿真结果如图 4 9 所示。

\section{表 2 仿真信号}

\begin{tabular}{ccc}
\hline 故障类型 & 一级 & 二级 \\
\hline & 30000 & 25000 \\
& 30000 & 20000 \\
执行机构弹簧疲劳/ $(\mathrm{N} / \mathrm{m})$ & 30000 & 15000 \\
& 25000 & 30000 \\
& 20000 & 30000 \\
& 15000 & 30000 \\
\hline 控制阀动作滞后/ms & 0 & -3 \\
& 0 & -5 \\
& 0 & -8 \\
& 0 & 3 \\
& -3 & 0 \\
耦合故障 & -5 & 0 \\
& -8 & 0 \\
& 25000 & 0 \\
\hline
\end{tabular}

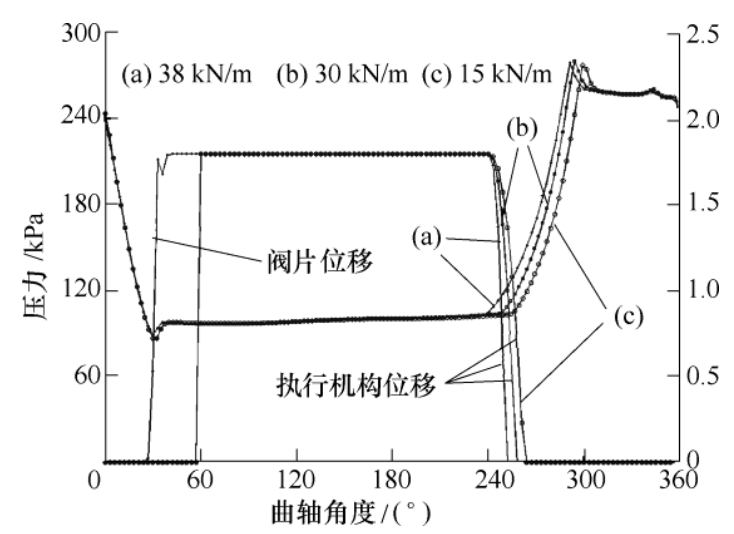

图 4 一级不同复位弹簧刚度的阀片、执行机构 位移与动态压力

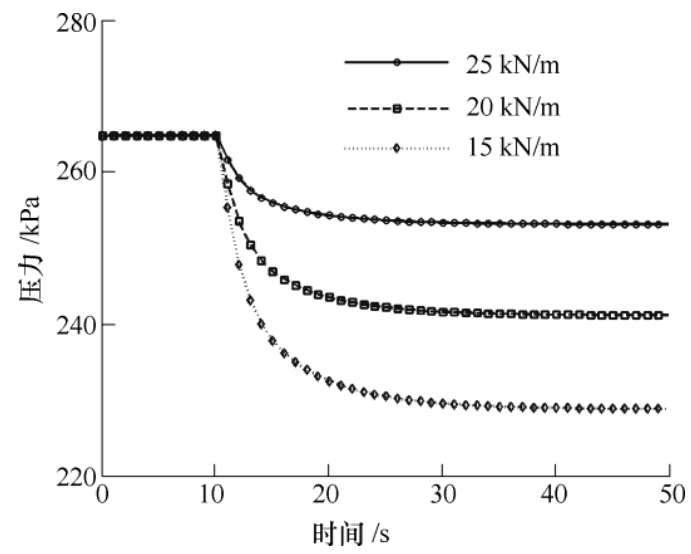

图 5 一级不同复位弹簧的一级缓冲罐压力变化

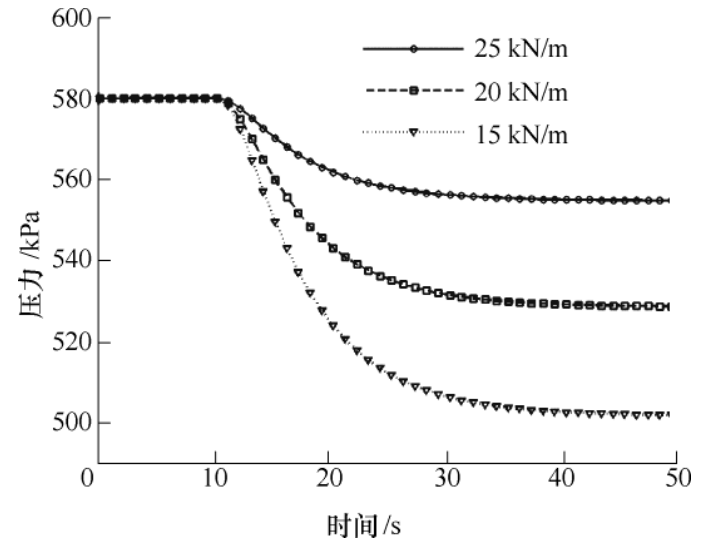

图 6 一级不同复位弹簧的二级缓冲罐压力变化

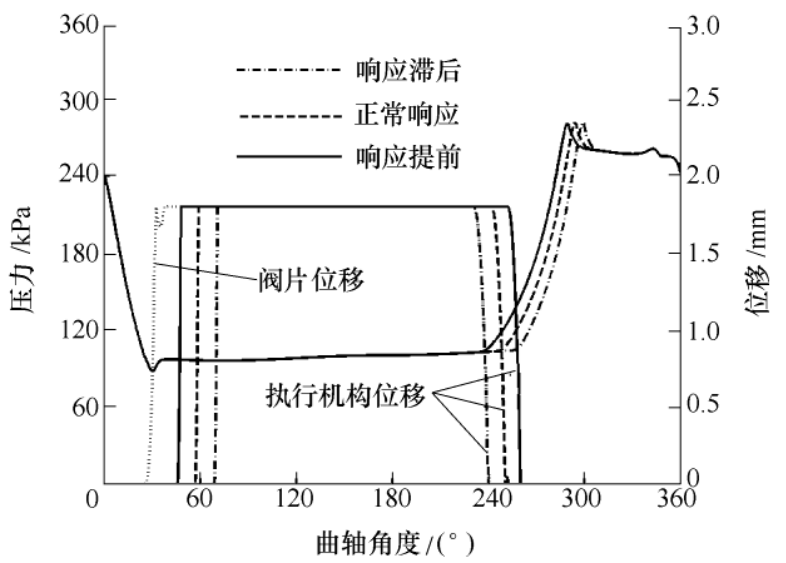

图 7 一级控制阀响应偏移, 阀片、执行机构位移与动态压力

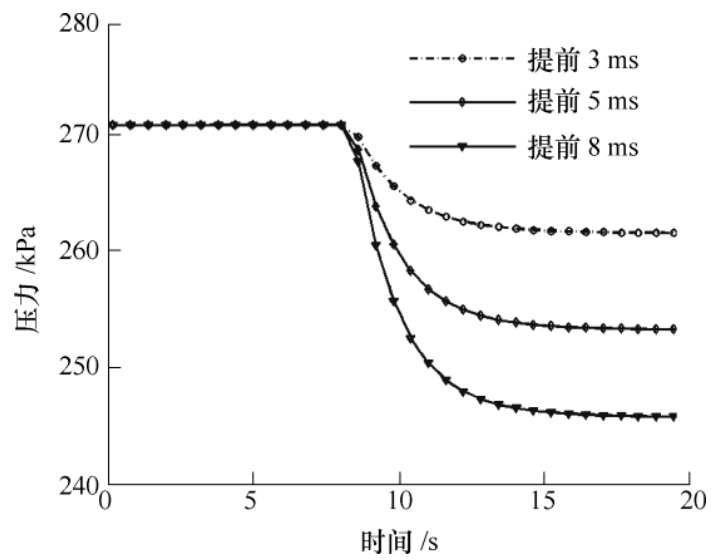

图 8 一级级控制阀响应提前, 一级缓冲罐压力变化

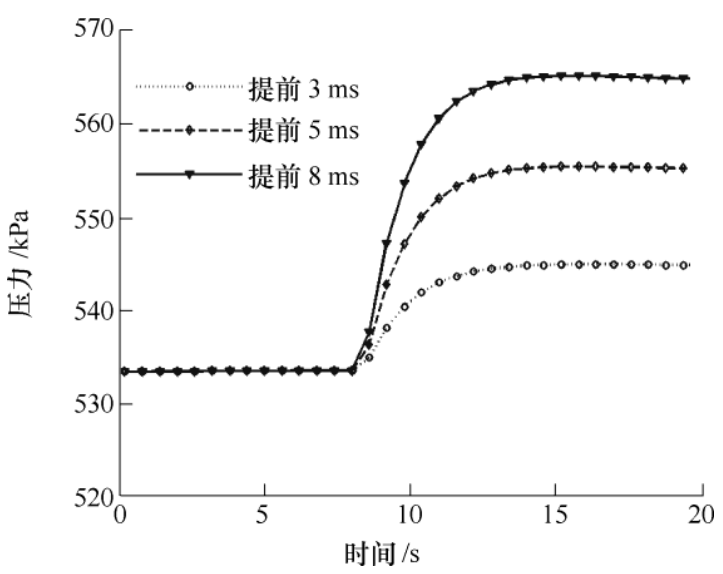

图 9 二级控制阀响应提前, 二级缓冲罐压力变化 
(1) 由图 4、图 7 可以看出, 弹簧刚度和控制阀 变化均会导致吸气阀阀片关闭时间变化。弹簧刚度 的减小不会影响吸气阀开始关闭角度, 只是增加阀 片关闭动作时间; 控制阀参数提前或者滞后, 不会 影响吸气阀关闭动作时间, 吸气阀开始关闭的角度 随着控制阀参数变化发生平移。

(2) 由图 5、6 可以看出, 一级弹簧刚度降低, 导致一级气体回流量增加, 使二级吸气量降低, 最 终导致一级/二级缓冲罐压力的下降。随着弹簧刚度 的减小, 压力下降的幅度增大, 因此弹簧刚度与压 力的关系是非线性的。

(3) 由图 8、9 可以看出, 二级控制阀响应提前, 导致二级气体回流量减小, 使二级吸气量增加, 导 致二级缓冲罐压力上升; 由于二级回流量的减少, 导致一级缓冲罐压力下降。由于吸气阀开始关闭的 角度随着控制阀参数变化发生平移, 因此控制阀参 数的偏移量与压力变化是趋于线性的。

\section{3 负荷反馈优化模型与自愈方案}

通过多系统耦合数学模型可以得到失稳参数与 压力的关系。除了压力参数外, 实际负荷还需要温 度、流量参数, 但是实际负荷与压力、温度、流量 是非线性关系, 且有扰动项 $\left(\mu_{i}\right)$, 如式(13)所示, 需 要构建 BP 神经网络, 通过历史数据训练, 预测负 荷值。确定机组负荷与调控目标参数的差值, 以差 值作为反馈值, 计算执行机构撤回角度修正量, 完 成自愈调控

$$
\eta_{i}=f\left(p_{i}, T_{i}, Q\right)+\mu_{i}
$$

\section{1 动态反馈优化模型}

BP 神经网络是一种误差反向传播神经网络, 是 人工神经网络的核心部分, 在模式识别、故障检测 和线性拟合等领域广泛应用。本文通过各级进排气 压力 $P_{\mathrm{i}}$ 、进排气温度 $T_{\mathrm{i}}$ 和机组流量 $\mathrm{Q}$ 可以构建多参 数神经网络反馈优化模型, 通过反馈优化模型对压 缩机各级气缸工作的实际状态进行实时评估, 得到 各级实际负荷的反馈估计值 $\tilde{\eta}_{i}\left(0 \leqslant \tilde{\eta}_{i} \leqslant 1\right)$ 。 $\mathrm{BP}$ 神 经网络反馈模型结构图如图 10 所示。

输入层包括几个主要参数: 各级进排气压力 $P_{\mathrm{i}}$; 各级进排气温度 $T_{\mathrm{i}}$; 机组的排气量 $Q$ 。假定机 组为 2 级压缩的机组, 由负荷反馈模型应用要求确 定输入层的神经元个数为 5 。

隐含层节点个数选取是一个特别复杂的问题, 隐含层节点个数过多或过少都会对网络带来影响, 导致网络训练时间增加或网络泛化能力不足。隐含

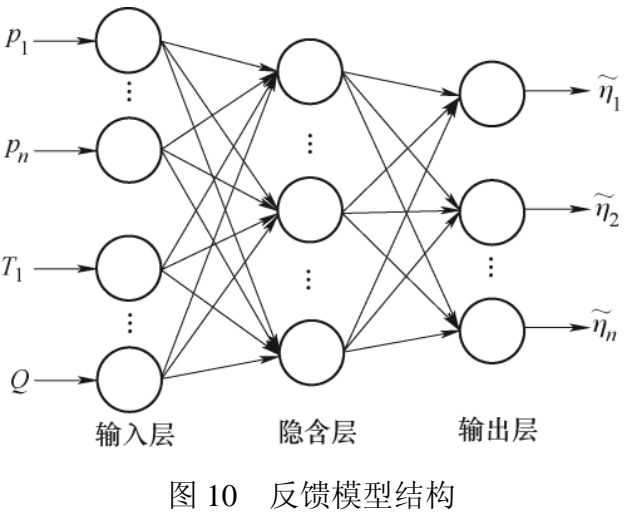

层节点数的确定目前尚缺少专业的理论指导, 根据 经验可参照式(14)进行设计

$$
M=\sqrt{m+n}+a
$$

式中, $M$ 为隐含层节点数; $m$ 为输入节点数; $n$ 为 输出节点数; $a$ 为 $0 \sim 10$ 之间的调节常数。通过上 式确定的隐含层结点范围为 $3 \sim 13$, 结合实际训练 效果确定, 当隐含层节点个数为 9 时, 此时效果最 佳。因此, 隐含层确定为 1 层, 隐含层神经元个数 为 9 。

输出层的输出结果为各级负荷实际的估计值, 因此, 需要 2 个输出层节点。

\section{2 失稳故障诊断与故障类型识别方法}

利用实际负荷反馈估计值计算控制系统输出各 级的控制负荷与实际负荷的偏差。

$$
\Delta \eta_{i}=\eta_{i}-\tilde{\eta}_{i}
$$

当负荷偏差 $\left|\Delta \eta_{i}\right| \leqslant \phi$, 可认为负荷调节是处于 正常状态, 其中 $\phi$ 取值为 0.03 表示负荷正常波动系 数, 这是由于系统从信号采集到控制信号输出累计 误差造成的。

当负荷偏差 $\left|\Delta \eta_{i}\right|>\phi$ 时, 致使压缩机的压比改 变, 影响压缩机的工作性能, 造成气量调节控制失 稳故障。为了进一步分析故障的原因, 通过振动信 号对执行机构动作进行实时监测。令执行机构复位 动作的振动冲击相位为 $\hat{\theta}_{i}$, 振动幅值为 $v_{a}$, 给定负 荷 $\eta$ 的预设复位相位为 $\theta_{i}$, 振动幅值为 $v_{b}$, 令执行 机构复位相位偏差引起的负荷偏差为 $\Delta \eta_{j}$, 振动幅 值偏差为 $\Delta v$ ，通过对比 $\Delta \eta_{j} 、 \Delta \eta_{i}$ 与 $\Delta v$ 可以实现 故障类型的识别。

\section{3 基于故障异常类型识别的气量调节控制失稳 故障自愈控制策略}

自愈的目标是实现气量调节系统的精确气量控 制, 保证调节系统的精度。从完成系统既定功能角 度, 把故障自愈系统结构分作自愈决策层、自愈执 行层。自愈决策层: 通过比对系统自身信息库中的 
事实和数据, 实时评估系统运行状态。判断故障自 愈请求, 制定自愈策略并寻找出最优方案。自愈执 行层: 执行相关系统指令。

往复压缩机气量调控自愈控制的流程图如 图 11 所示。

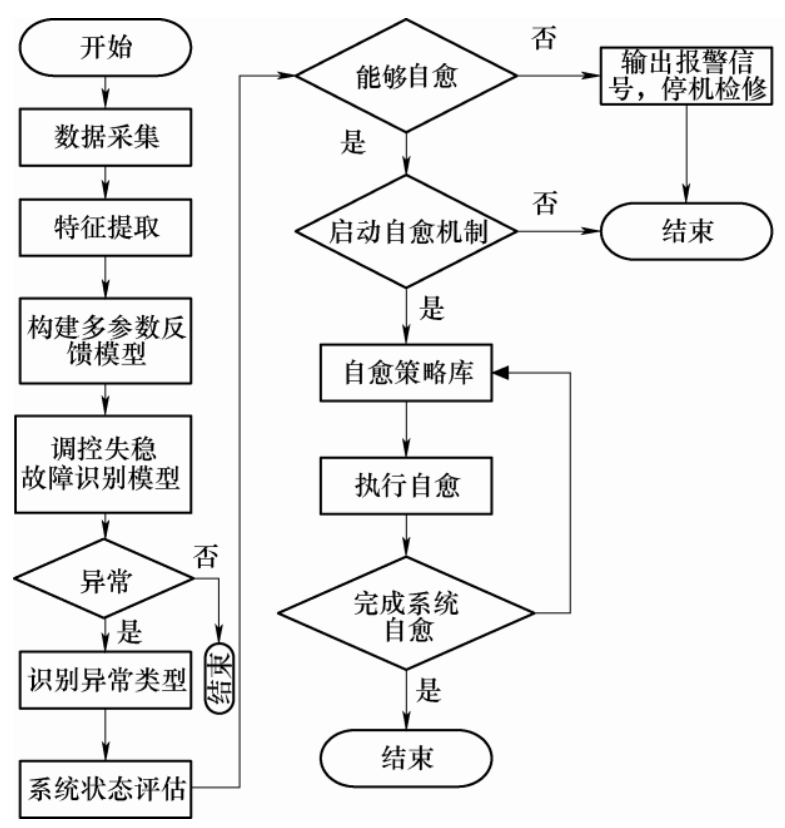

图 11 调控失稳故障自愈流程

自愈决策层通过反馈估计模型和振动信号对比 分析对往复压缩机无级气量调节系统调节运行状态 进行实时评估, 结果采用二进制对系统运行状态进 行编码, 见表 3 。

表 3 调节系统调节效果状态编码

\begin{tabular}{ccc}
\hline 序号 & 系统调节状态类型 & 状态编码 \\
\hline 1 & 正常状态 & 00 \\
2 & 复位弹簧疲劳 & 01 \\
3 & 控制阀参数偏移 & 10 \\
4 & 多个故障耦合 & 11 \\
\hline
\end{tabular}

本文提出的自愈控制方案如下。

(1) 系统调节状态类型为正常状态, 即状态编 码为 00 , 停止启动自愈机制。

(2) 系统状态编码为 01 时, 首先对性能参数改 变的执行机构进行定位, 可具体定位到压缩机某一 级某一侧(压缩机的盖侧或者轴侧)执行机构, 假设 发生性能参数变化的执行机构在第 $k$ 级盖侧, 通过 振动信号反馈得出实际执行机构撤回的角度偏离当 前负荷的设计角度变化量为 $\Delta \theta_{k}$, 建立执行机构性 能参数变化量与脉宽调制信号的调整量之间的关系 $f_{1}$, 通过此关系计算施加到第 $k$ 级盖侧执行机构的
调控参数脉宽调制信号的调整量 $\Delta T_{k}$ 。

$$
\Delta T_{k}=f_{1}\left(\Delta \theta_{k}\right)=\alpha \frac{\left(\theta_{k}-\hat{\theta}_{k}\right)}{\omega}
$$

式中， $\alpha$ 为自愈系数， $\theta_{k}$ 为当前控制负荷的设计撤 回角度, $\hat{\theta}_{k}$ 为振动信号反馈角度, $\omega$ 为压缩机曲轴 转动的角速度。

(3) 系统状态编码为 10 时, 首先对发生泄漏的 位置进行定位, 泄漏只能定位到某一级, 假设发生 性能参数变化的执行机构在第 $m$ 级, 通过反馈模型 获取实际负荷与当前控制负荷的偏差量 $\Delta \eta_{m}$, 建立 负荷偏差量与脉宽调制信号的调整量之间的关系 $f_{2}$, 脉宽调制信号的调整量为 $\Delta T_{m}=f_{2}\left(\eta_{m}, \Delta \eta_{m}\right)$, 施 加到第 $m$ 级上所有执行机构的调控参数中，同时对 该位置不同负荷对应的执行机构撤回的设计角度增 加修正量 $\Delta \theta_{m}$ 。

$$
\begin{gathered}
\Delta T_{m}=\beta \frac{1}{\omega}\left[\arccos \left(\frac{1}{\lambda}-\sqrt{1+\frac{2}{\lambda}-\frac{4 \eta_{m}}{\lambda}+\frac{1}{\lambda^{2}}}\right)-\right. \\
\left.\arccos \left(\frac{1}{\lambda}-\sqrt{1+\frac{2}{\lambda}-\frac{4\left(\eta_{m}-\Delta \eta_{m}\right)}{\lambda}+\frac{1}{\lambda^{2}}}\right)\right] \\
\Delta \theta_{m}=f_{1}\left(\Delta \theta_{k}\right)=\theta_{m}+2 \pi \times \Delta T_{m} / \omega
\end{gathered}
$$

式中, $\beta$ 为自愈系数, $\eta_{m}$ 为当前控制负荷, $\lambda$ 为压 缩机曲轴连杆比。

(4) 系统状态编码为 11 时, 实施自愈控制分两 步, 第一步, 对执行机构性能参数变化进行自愈控 制; 第二步，对泄漏进行自愈控制。首先对故障的 执行机构进行定位，假设发生故障的执行机构在第 $n$ 级, 其中执行机构性能参数变化的为轴侧, 通过 振动信号反馈得出实际执行机构撤回的角度偏离当 前负荷的设计角度量为 $\Delta \theta_{n}$, 按照式(17)计算出轴侧 脉宽调制信号的调整量 $\Delta T_{1 n}$; 其次, 施加调整量后, 利用反馈模型计算负荷的偏差量 $\Delta \eta_{n}$, 按照式(18) 计算出第 $n$ 级脉宽调制信号的调整量 $\Delta T_{2 n}$, 并对应 位置的不同负荷对应的执行机构撤回的设计角度按 照式(18)进行修正。

\section{4 试验验证}

为研究两级往复压缩机无级气量调节系统 失稳自愈调控方法, 在往复压缩机试验平台上进 行了试验研究, 包括压缩机、液压站、控制系统、 执行机构等组件, 如图 12 所示。试验台参数如 表 4 所示。 


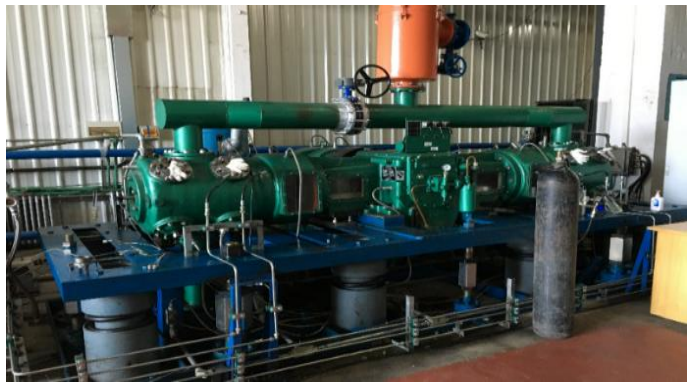

图 12 气量调节系统试验台

表 4 机组与调节系统主要参数表

\begin{tabular}{|c|c|c|c|}
\hline 参数 & 数值 & 参数 & 数值 \\
\hline $\begin{array}{l}\text { 额定转速 } \\
/(\mathrm{r} / \mathrm{min})\end{array}$ & 300 & $\begin{array}{l}\text { 吸气压力 } \\
/ \mathrm{kPa}\end{array}$ & 100 \\
\hline 吸气阀数量 & $4 / 4$ & $\begin{array}{l}\text { 液压缸 } \\
\text { 直径/mm }\end{array}$ & 10 \\
\hline 气缸数 & 2 & $\begin{array}{l}\text { 一级缓冲罐 } \\
\text { 容积 } / \mathrm{m}^{3}\end{array}$ & 0.3 \\
\hline 流量 $/\left(\mathrm{m}^{3} / \mathrm{h}\right)$ & 12 & $\begin{array}{l}\text { 二级缓冲罐 } \\
\text { 容积 } \mathrm{m}^{3}\end{array}$ & 0.16 \\
\hline $\begin{array}{l}\text { 复位弹簧 } \\
\text { 刚度/(kN/m) }\end{array}$ & 30 & 余隙容积系数 & $0.125 / 0.125$ \\
\hline 曲柄连杆比 & 0.2 & 排气压力/kPa & $250 / 550$ \\
\hline
\end{tabular}

在执行机构内部安装位移传感器, 可以测量吸 气阀阀片的动态响应过程; 在压缩机气缸示功孔上
安装压力传感器, 可以测量气缸动态压力; 在压缩 机缸头和缸体上安装加速度传感器, 可以测量振动; 在缓冲罐上安装压力传感器, 可以测量缓冲罐压力。 部分传感器布置如图 13 所示。

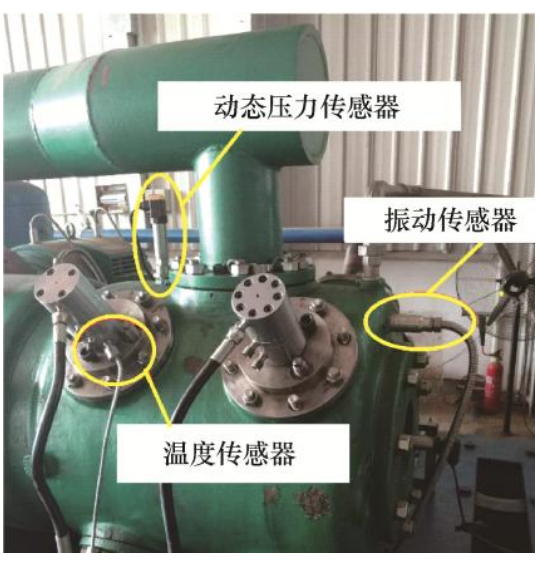

图 13 往复压缩机试验台传感器布置

通过设置两级不同的压力, 记录机组稳定时的 温度、流量及负荷数据, 为 $\mathrm{BP}$ 神经网络积累训练 数据。试验数据如表 5 所示, 由于数据较多, 只列 出压力数据。表 6 为 $\mathrm{BP}$ 神经网络训练结果, 从训 练的结果看, 误差均小于 $2 \%$, 具有很好的预测性。

表 5 BP 训练数据

\begin{tabular}{|c|c|c|c|c|c|c|c|c|c|c|}
\hline 编号 & 1 & 2 & 3 & 4 & 5 & 6 & 7 & 8 & 9 & 10 \\
\hline 一级排气压力 $/ \mathrm{kPa}$ & 229 & 234 & 237 & 228 & 216 & 218 & 221 & 223 & 224 & 222 \\
\hline 二级排气压力 $/ \mathrm{kPa}$ & 217 & 264 & 316 & 362 & 406 & 454 & 502 & 534 & 570 & 548 \\
\hline 编号 & 11 & 12 & 13 & 14 & 15 & 16 & 17 & 18 & 19 & 20 \\
\hline 一级排气压力 $/ \mathrm{kPa}$ & 219 & 209 & 232 & 256 & 284 & 315 & 343 & 392 & 353 & 329 \\
\hline 二级排气压力 $/ \mathrm{kPa}$ & 518 & 474 & 488 & 502 & 513 & 523 & 526 & 518 & 533 & 534 \\
\hline 编号 & 21 & 22 & 23 & 24 & 25 & 26 & 27 & 28 & 29 & 30 \\
\hline 一级排气压力 $/ \mathrm{kPa}$ & 296 & 309 & 277 & 251 & 247 & 246 & 248 & 218 & 188 & 158 \\
\hline 二级排气压力 $/ \mathrm{kPa}$ & 535 & 498 & 501 & 498 & 458 & 424 & 390 & 381 & 358 & 326 \\
\hline 编号 & 31 & 32 & 33 & 34 & 35 & 36 & 37 & 38 & 39 & 40 \\
\hline 一级排气压力 $/ \mathrm{kPa}$ & 140 & 161 & 181 & 210 & 252 & 273 & 297 & 297 & 282 & 286 \\
\hline 二级排气压力 $/ \mathrm{kPa}$ & 272 & 288 & 338 & 348 & 346 & 290 & 300 & 285 & 349 & 389 \\
\hline
\end{tabular}

表 6 BP 神经网络测试表格

\begin{tabular}{ccccc}
\hline 编号 & 输入 $/ \mathrm{kPa}$ & 训练结果 $(\%)$ & 目标值 $(\%)$ & 差值 \\
\hline 1 & $253 / 554$ & $79.5 / 81.0$ & $80 / 80$ & $0.5 / 1$ \\
2 & $270 / 532$ & $81.4 / 90.6$ & $80 / 90$ & $1.4 / 0.6$ \\
3 & $236 / 572$ & $80.3 / 71.2$ & $80 / 70$ & $0.3 / 1.2$ \\
4 & $281 / 522$ & $78.2 / 66.1$ & $80 / 65$ & $1.8 / 1.1$ \\
\hline
\end{tabular}

根据三种故障类型, 采用不同的试验参数进行
试验, 验证自愈控制的可行性, 试验参数如表 7 所 示。不同故障类型下，自愈调控效果见表 8 。

\section{表 7 试验参数}

\begin{tabular}{ccc}
\hline 故障类型 & 控制阀性能偏移 $/ \mathrm{ms}$ & 弹簧疲劳 $/(\mathrm{N} / \mathrm{m})$ \\
\hline 01 & 控制阀正常 & 弹簧刚度 20000 \\
10 & 二级控制阀提前增加 6 & 弹簧正常 \\
11 & 二级控制阀提前增加 6 & 弹簧刚度 20000 \\
\hline
\end{tabular}


表 8 自愈调控参数

\begin{tabular}{|c|c|c|c|c|c|c|c|}
\hline 故障类型 & $\begin{array}{c}\text { 给定 } \\
\text { 负荷值 }(\%)\end{array}$ & $\begin{array}{c}\text { 阀体 } \\
\text { 温度/ }{ }^{\circ} \mathrm{C}\end{array}$ & 压力/kPa & $\begin{array}{c}\text { 反馈 } \\
\text { 负荷值(\%) }\end{array}$ & $\begin{array}{l}\text { 复位振动 } \\
\left.\text { 相位 } /^{\circ}{ }^{\circ}\right)\end{array}$ & $\begin{array}{l}\text { 调控参数 } \\
\text { 调整量/ms }\end{array}$ & $\begin{array}{c}\text { 故障恢复后 } \\
\text { 压力/kPa }\end{array}$ \\
\hline 00 & $80 / 80$ & $39.8 / 40.2$ & $253 / 554$ & $79.5 / 81.0$ & $241 / 239$ & $0 / 0$ & $253 / 554$ \\
\hline 01 & $80 / 80$ & $40.2 / 30.1$ & $270 / 532$ & $81.4 / 90.6$ & $239 / 229$ & $0 / 4.5$ & $250 / 550$ \\
\hline 10 & $80 / 80$ & $40.7 / 50$ & $236 / 572$ & $80.3 / 71.2$ & $242 / 250$ & $0 /-4.3$ & $254 / 555$ \\
\hline 11 & $80 / 80$ & $40 / 40$ & $281 / 522$ & $78.2 / 66.1$ & $243 / 258$ & $0 /-7.6$ & $252 / 558$ \\
\hline
\end{tabular}

以控制阀动态特性参数偏移的自愈效果为例, 分析模型的正确性和调控的可行性。调控效果如图 14 17 所示。从图 14 和图 15 可以看出, 系统在 A 时刻处于正常工作状态, 一级和二级排气压力分别 稳定在 $253 \mathrm{kPa}$ 和 $554 \mathrm{kPa}$, 在 $\mathrm{B}$ 时刻二级换上动态 参数滞后的控制阀, 一级排气压力快速升高至 $270 \mathrm{kPa}$, 二级排气迅速下降至 $532 \mathrm{kPa}$, 在 $\mathrm{C}$ 时刻 启动自愈调控 $\mathrm{X}$ 机制, 排气压力快速下降最终稳 定, 在自愈完成 $\mathrm{D}$ 时刻一级和二级排气压力分别 为 $250 \mathrm{kPa}$ 和 $556 \mathrm{kPa}$, 自愈调控曲线与仿真值基 本重合, 验证了仿真的可行性。图 16 表示一级执 行机构动作的振动相位, 一级执行机构在 $A 、 B$

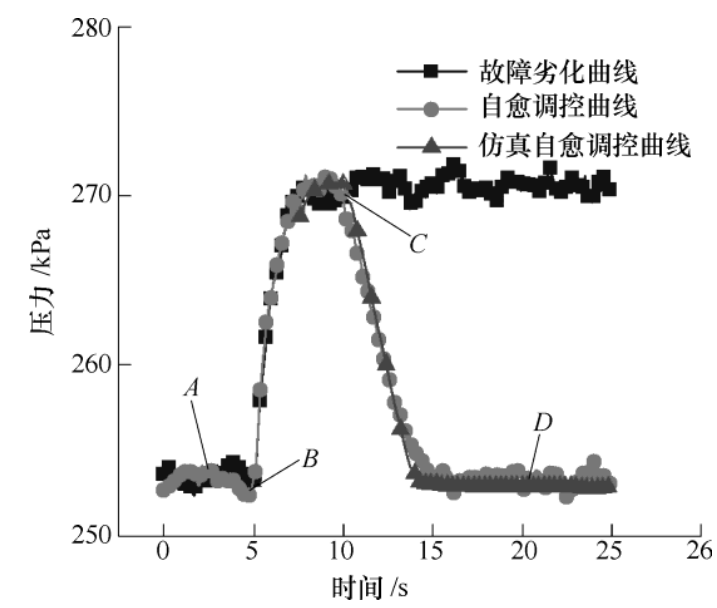

图 14 自愈调控制一级压力趋势图

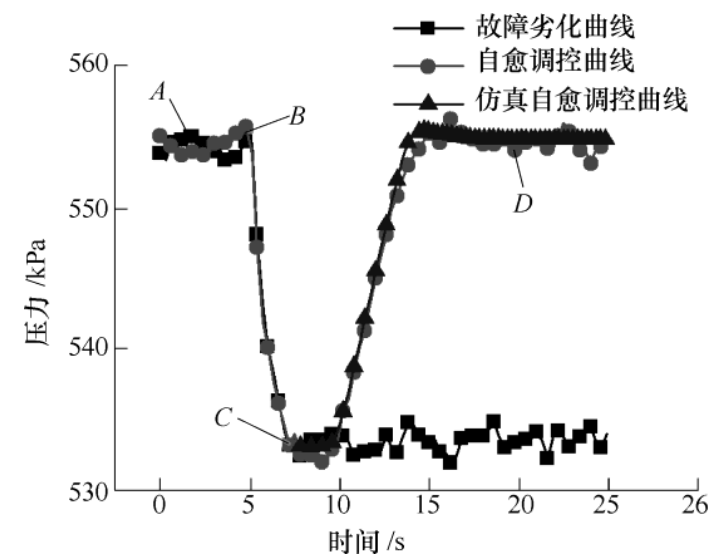

图 15 自愈调控制二级压力趋势图
和 $D$ 时刻的振动相位几乎不变, 说明一级调节的 负荷是稳定的, 没有出现故障。图 17 表示二级执 行机构动作的振动相位, 在点 $B$ 时刻振动相位角 度向后出现了较大整体向后平移, 且振动幅值基 本不变, 可以判定是二级控制阀动态参数滞后的 失稳故障。

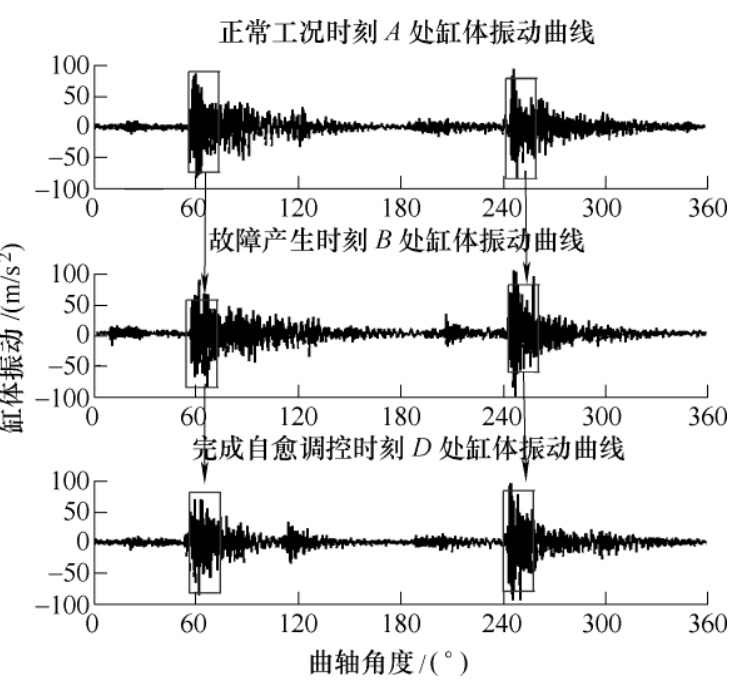

图 16 自愈调控制不同时刻一级缸体振动图

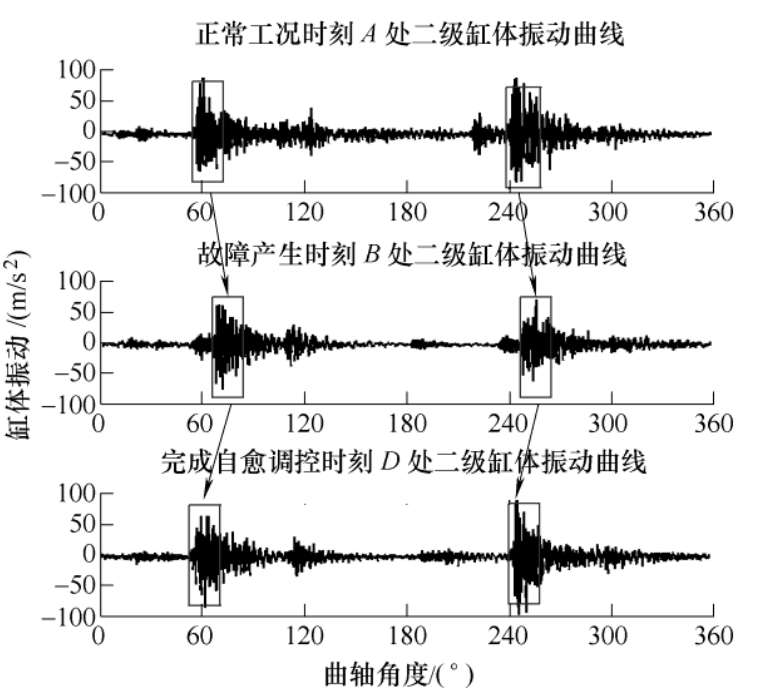

图 17 自愈调控制不同时刻二级缸体振动图 


\section{5 结论}

针对往复压缩机气量调节失稳的问题, 首先通 过建立的多系统耦合控制模型研究了执行机构动态 响应和控制系统调控参数对气量调节效果的影响规 律，通过改变控制信号占空比信号实现气量无级控 制。仿真结果得出: 控制系统调控参数和执行机构 的动态响应变化影响气量调节效果。

（1）执行机构复位弹簧刚度对气量调节的影 响: 撤回的动作时间随着复位弹簧刚度降低而增加。 在相同的控制信号(相同负荷控制)下，弹簧刚度下 降, 执行机构撤回时间变长, 导致阀片完全关闭的 相位延迟增大, 实际负荷相较于给定的控制负荷偏 低。造成前后端缓冲罐压力下降。

(2) 控制阀动态特性偏移, 使系统调控参数与 控制阀标定的性能特性参数下的预设调控参数失 配, 造成机组负荷调控精度下降, 随着控制阀动态 特性变化加剧, 造成机组级间缓冲罐压力超过其安 全范围或者使末级压比超高, 最终造成气量调控失 稳的故障。

本文利用 BP 神经网络构建的负荷反馈模型提 出了失稳故障和故障类型识别诊断方法, 基于失稳 故障类型进一步地提出了自愈调控方案, 试验结果 表明提出的自愈调控方案可根据失稳故障的类型在 线自适应调整优化补偿系统调控参数实现故障自 愈。使得机组在不停机的前提下, 实现机组由气量 调节失稳状态恢复到正常调节状态, 确保了压缩机 的健康稳定运行, 解决了停车损失, 维修成本居高 不下, 并且系统过于依赖人工操作的问题, 系统安 全性及可靠性提高。

\section{参 考文 献}

[1] 刘广森, 唐斌, 赵远扬, 等. 往复压缩机流量无级调节 系统动态特性研究[J]. 机械工程学报, 2017, 53(23):

26-31.

LIU Guangbin, TANG Bin, ZHAO Yuanyang, et al. Dynnamic performance of reciprocating compressor with capacity stepless regulation system[J]. Journal of Mechanical Engineering, 2017, 53(23): 26-31.

[2] BIN T, YUANYANG Z, LIANSHENG L, et al. Thermal performance analysis of reciprocating compressor with stepless capacity control system[J]. Applied Thermal Engineering, 2013, 54(2): 380-386.

[3] GU Z L, HOU X P, WANG Z S, et al. Methods for large reciprocating compressor capacity control: A review based on pulse signal concept[J]. Chinese Science Bulletin, 2011(19): 5-12.

[4] XIN WANG. Hoerbiger smart control\&monitoring system-hydrocom system[C]// Proceedings of the 3rd International Compressor Technique Conference. Xi'an: Xi'an Jiaotong University, 2001: 348-353.

[5] 洪伟荣, 叶林. 无级气量调节对往复式压缩机的力学影 响[J]. 工程热物理学报, 2011, 32(supplement 1): 49-52. HONG Weirong, YE Lin. The mechanical effects of reciprocating compressor in full-range capacity stepless control system[J]. Journal of Engineering Thermophysics, 2011, 32(Suppl. 1): 49-52.

[6] 王蒙, 孙旭, 张进杰. 往复压缩机气量无级调节工况下 吸气阀故障模拟与诊断 $[\mathrm{J}]$. 流体机械，2019，47(4): 39-44.

WANG Meng, SUN Xu, ZHANG Jinjie. Fault simulation and diagnosis of suction valve on reciprocating compressor with stepless capacity control[J]. Fluid Machinery, 2019, 47(4): 39-44.

[7] 郑诏星, 江志农, 王瑶, 等. 往复压缩机无级气量调节 系统卸荷器动作特性研究 [J]. 机械强度, 2019, 41(3): 238-242.

ZHENG Zhaoxing, JIANG Zhinong, WANG Yao, et al. Study on the operating characteristics of unloader of reciprocating compressor stepless capacity regulation system[J]. Journal of Mechanical Strength，2019，41(3): 238-242.

[8] 盛尊祥, 赵永兴, 孟玉清, 等. 无级气量调节系统卸荷 杆填料密封失效的研究与对策 $[\mathrm{J}]$. 压缩机技术, 2013, 5: 66-68.

SHENG Zunxiang, ZHAO Yongxing, MENG Yuqing, et al. Research of packing seal failure occurred in unloading rod of stepless capacity control system and countermeasures [J]. Compressor Technology, 2013, 5: 66-68.

[9] 吴荣仁, 管宇辉. 全量程可调气阀的调节机构和最优调 节力的分析 $[\mathrm{J}]$. 流体机械，2005，33(6)：22-25.

WU Rongren, GUAN Yuhui. Regulation device in infinitely variable capacity valve and analysis on optimized regulation force[J]. Fluid Machinery, 2005, $33(6): 22-25$

[10] 李木林, 赵广友, 洪伟荣, 等. 往复压缩机气量调节工 况阀片侧倾分析 [J]. 压缩机技术，2013，1：14-16.

LI Mulin, ZHAO Guangyou, HONG Weirong, et al. 
Valve plate roll analysis on stepless capacity regulation for reciprocating compressor[J]. Compressor Technology, 2013, 1: 14-16.

[11] 曹继来, 李颖, 吴价, 等. 压缩机气量调节系统液压执行 机构性能的研究[J]. 机床与液压, 2015，43(23)：35-38.

CAO Jilai, LI Ying, WU Jia, et al. Study of hydraulic actuator performance used in compressor capacity regulation system[J]. Machine Tool \& Hydraulics, 2015, 43(23): $35-38$.

[12] LI Yun, YANG S H. Research on the fault diagnosis and self-healing technology of unattended Antarctic telescope[C]// Ground-based and Airborne Telescopes VII, July 6, 2018, Austin, Texas, United States. SPIE, 2018: 107004W.

[13] GOMEZ-ANDRADES A , LUENGO P M , KHATIB E, et al. Methodology for the design and evaluation of self-healing LTE networks[J]. IEEE Transactions on Vehicular Technology, 2015, 65(8): 6468-6486.

[14] BUTLERPURRY K L, SARMA N D. Self-healing reconfiguration for restoration of naval shipboard power systems[J]. IEEE Transactions on Power Systems, 2004, 19(2): 754-762.

[15] 刘凯, 李文佩, 程潜善, 等. 基于启发式规则和人工免疫 的智能配电网自愈 $[J]$. 武汉大学学报, 2016, 49(2): 218-222.

LIU Kai, LI Wenpei, CHENG Qianshan, et al. Self-healing of smart distribution network based on heuristic rules and artificial immune[J]. Engineering Journal of Wuhan University, 2016，49(2): 218-222.

[16] 高金吉. 机器故障诊治与自愈化 $[\mathrm{M}]$. 北京：高等教育 出版社, 2012.

GAO Jinji. Machines fault diagnosis and self-recovery[M]. Beijing: Higher Education Press, 2012.

[17] 李启行, 王维民, 刘宾宾, 等. 基于电磁激励的柔性转 子系统轴承等效参数辨识 $[\mathrm{J}]$. 机械工程学报, 2017, 53(23): 1-6.

LI Qihang, WANG Weimin, LIU Binbin, et al. Electromagnetic-excitation-based identification method for identical rotor-bearings system parameters[J]. Journal of Mechanical Engineering, 2017, 53(23): 1-6.

[18］孙文理, 袁宇浩, 袁野. 电液伺服系统的故障自愈设计 与仿真 $[J]$. 计算机工程与设计, 2018, 39(6): 1716-1720. SUN Wenli, YUAN Yuhao, YUAN Ye. Design and simulation of fault self-healing for electro-hydraulic servo system[J]. Computer Engineering \& Design, 2018, 39(6): 1716-1720.

作者简介: 江志农, 男, 1967年出生, 教授, 博士研究生导师。主要研 究方向为关键设备故障监测诊断与性能分析等。

E-mail: jiangzhinong@263.net

周超, 男, 1991年出生, 博士。主要研究方向为往复机械气量调节与故 障诊断。

E-mail: 15117950620@163.com

张进杰(通信作者), 男, 1987 年出生, 博士, 副教授。主要研究方向为 设备故障诊断机理与诊断方法智能化。

E-mail: zjj87427@163.com 